\title{
The role of lung ultrasound as a frontline diagnostic tool in the era of COVID-19 outbreak
}



 \\ the Fenice Network (Italian Group for Clinical Research in Emergency Medicine)
}

Received: 1 June 2020 / Accepted: 29 September 2020 / Published online: 22 October 2020

(c) Società Italiana di Medicina Interna (SIMI) 2020

\begin{abstract}
The diffusion of the severe acute respiratory syndrome coronavirus-2 (SARS-CoV-2) worldwide prompted the World Health Organization to declare the status of pandemic. The molecular diagnosis of SARS-CoV-2 infection is based on the detection of viral RNA on different biological specimens. Unfortunately, the test may require several hours to be performed. In the present study, we evaluated the diagnostic accuracy of lung point-of-care ultrasound (POCUS) for SARS-CoV-2 pneumonia in a cohort of symptomatic patients admitted to one emergency department (ED) in a high-prevalence setting. This retrospective study enrolled all patients who visited one ED with suspected respiratory infection in March 2020. All the patients were tested (usually twice if the first was negative) for SARS-CoV-2 on ED admission. The reference standard was considered positive if at least one specimen was positive. If all the specimens tested negative, the reference was considered negative. Diagnostic accuracy was evaluated using sensitivity, specificity, and positive and negative predictive value. Of the 444 symptomatic patients who were admitted to the ED in the study period, the result of the lung POCUS test was available for 384 (86.5\%). The sensitivity of the test was $92.0 \%$ (95\% CI 88.2-94.9\%), and the specificity was $64.9 \%$ (95\% CI 54.6-74.4\%). We observed a prevalence of SARS-CoV-2 infection of 74.7\%. In this setting, the positive and negative predicted values were 88.6\% (95\% CI 84.4-92.0) and 73.3\% (95\% CI 62.6-82.2\%), respectively. Lung POCUS is a sensitive first-line screening tool for ED patients presenting with symptoms suggestive of SARS-CoV-2 infection.
\end{abstract}

Keywords COVID-19 $\cdot$ SARS-CoV-2 $\cdot$ Point-of-care ultrasound $\cdot$ Diagnostic test $\cdot$ Sensitivity $\cdot$ Specificity

\section{Introduction}

Electronic supplementary material The online version of this article (https://doi.org/10.1007/s11739-020-02524-8) contains supplementary material, which is available to authorized users.

Giovanni Nattino giovanni.nattino@marionegri.it

1 Accident and Emergency Services, ASST Santi Paolo e Carlo, Via Pio II 3, 20153 Milan, Italy

2 Department of Advanced Diagnostic-Therapeutic Technologies, ASST Santi Paolo e Carlo, Via Pio II 3, 20153 Milan, Italy

3 Laboratory of Clinical Epidemiology, Department of Public Health, Istituto di Ricerche Farmacologiche Mario Negri IRCCS, Via G.B. Camozzi 3, 24020 Ranica, BG, Italy
Coronavirus disease 19 (COVID-19) represents a new challenge among infectious diseases. It was first described in early December 2019 in China [1] and, since then, it has quickly spread so much around the world that the World Health Organization has declared the status of pandemic on

4 Postgraduation School in Emergency Medicine, Università degli Studi di Milano, Via Festa del Perdono 7, 20122 Milan, Italy

5 Postgraduation School in Radiodiagnostics, Università degli Studi di Milano, Via Festa del Perdono 7, 20122 Milan, Italy

6 Postgraduation School in Internal Medicine, Università degli Studi di Milano, Via Festa del Perdono 7, 20122 Milan, Italy 
March 11th 2020 [2]. The infection is sustained by a novel coronavirus, soon named severe acute respiratory syndrome coronavirus-2 (SARS-CoV-2) because of the high rate of lung involvement in the infected population.

The disease has hit Italy hard, with the highest prevalence in Lombardy region, where different epidemic outbreaks have developed. In this context, emergency departments (EDs) were rapidly overwhelmed by the enormous number of patients, who had to be promptly managed. A simple, quick, and reliable diagnostic test to identify SARS-CoV-2 pneumonia is essential, so to immediately start the treatments and timely decide whether to admit the patient in a COVID-19-dedicated or COVID-19-free ward.

The molecular diagnosis of SARS-CoV-2 infection is based on the detection of viral RNA on different biological samples (commonly nasopharyngeal swabs), through reverse transcriptase polymerase chain reaction (RT-PCR). This test takes about $6 \mathrm{~h}$ to be performed [3], but its results may require up to $36 \mathrm{~h}$ to reach patients and healthcare practitioners when the samples have to be sent remotely or in the case of laboratory overload. The accuracy of such a test is still unclear, as false negative rates as high as $30 \%$ have been reported [3-10].

SARS-CoV-2 infection with pulmonary involvement shows typical computed tomography (CT) findings [3-6]. With a reported sensitivity of $94 \%$ and specificity of $37 \%$ $[11,12]$, CT scan is considered the most accurate, first-line imaging test to evaluate patients admitted to the ED with respiratory symptoms suggestive of SARS-CoV-2 infection. In these cases, CT scan is particularly useful if RT-PCR is negative.

Nevertheless, CT scan is time consuming, exposes patients to transport-related risks and to ionizing radiation. Moreover, during the pandemic, the unbalance between the available resources and the number of patients to manage makes CT scan very impractical as a systematic assessment.

Chest X-ray is the most frequently performed radiological examination in the ED, in particular among patients with respiratory symptoms, due to its wide availability, low cost, and low radiation exposure. It provides a global two-dimensional picture of lung parenchyma. Because of its limited spatial resolution and diagnostic accuracy, particularly in infectious interstitial pneumonia [13], chest X-ray plays a key role in the monitoring of patients' clinical course rather than in the diagnostic phase during a pandemic, in which a very sensitive test is necessary $[14,15]$.

Nowadays, lung point-of-care ultrasound (POCUS), performed and interpreted by emergency physicians, has a widespread use [16] and its role in the evaluation of patients with both cardiogenic and pneumogenic dyspnea is well established [17]. Previous studies have described the sonographic signs of COVID-19 pneumonia [18], which include B lines (hyperechoic, laser-like artifacts arising from the pleural line), often in a pattern known as interstitial syndrome (>3B-lines in more than 2 lung regions), an irregular or fragmented pleural line and peripheral lung consolidations. Interspersed areas of spared lung, similar to Acute Respiratory Distress Syndrome (ARDS), have been observed, mostly with preserved pleural sliding and in the absence of pleural effusion. The current pandemic has caused a significant increase in ED admissions of patients with respiratory symptoms. To improve the clinical and organizational workflow and reduce the risk to infect nonCOVID-19 patients, a quick and reliable differential diagnostic tool to distinguish between patients with suspected COVID-19 infection and those with symptoms secondary to other causes is essential.

In the present study, we aim at evaluating the accuracy of lung POCUS in the diagnosis of COVID-19-associated pneumonia, as compared to the result of RT-PCR test in symptomatic patients admitted to the ED in a high-prevalence setting.

To the best of our knowledge, this topic has not been previously investigated.

\section{Materials and methods}

\section{Patients selection}

This single-center, retrospective study enrolled all patients who consecutively visited the ED at San Carlo Borromeo Hospital in Milan, Italy, with signs and symptoms of respiratory infection, between March 1st and March 30th, 2020. Specifically, we considered all the patients presenting with cough, dyspnea, or a combination of these signs with fever.

Patients' characteristics were extracted from the ED and hospital electronic medical records. These characteristics included age, sex, signs, and symptoms on ED arrival and the results of POCUS test, chest X-ray, and CT scan, when performed. Although the POCUS test and chest X-ray were routinely performed on nearly all patients satisfying the eligibility criteria of this study (according to the current clinical practice of the center), chest CT scan was only performed out of clinician's decision, in case of diagnostic uncertainty.

The institutional review board provided ethical approval for the retrospective data collection, within the broader ECCOVID cohort study [19].

\section{Lung ultrasound assessment}

All the operators had received education in lung ultrasound (i.e., a single-day course on chest POCUS and additional hands-on training, including 150 exams under supervision) before the pandemic outbreak. Lung ultrasound was performed adopting the 12-region model, 6 on each side, 
dividing each hemithorax into an anterior, lateral, and posterior areas (delimitated by the anatomical landmarks represented by axillary lines), and each area into an upper and lower segment, as previously proposed by Via et al. [20].

All lung POCUS findings were reported in the electronic medical records, allowing the retrospective data collection. Lung POCUS was performed at the bedside just after the first clinical evaluation, and was considered positive for COVID-19 pneumonia in case of:

- Interstitial lung syndrome: two or more positive regions bilaterally (regions are defined as positive in presence of 3 or more B-lines on a longitudinal plane between two ribs) with irregular pleural line [21].

- Interstitial lung pattern: two or more positive regions with irregular pleural line, with focal/unilateral distribution.

- White lung (coalescent B lines) in two or more zones.

- Subpleural consolidations.

Significant pleural effusion alone was not considered a consistent finding for COVID-19 pneumonia [22].

\section{Chest X-ray}

All chest X-ray reports were reviewed and classified as positive or negative for pulmonary infection regardless the specific suspicion of lung involvement from SARS-CoV-2 infection. In case of ambiguous reports, the definitive decision was performed in a consensus reading between the author of the report and a radiologist with 5-year experience.

\section{Reference standard}

All the eligible patients underwent RT-PCR test on one or more respiratory specimens on ED arrival. Specimens included nasopharyngeal or oropharyngeal swabs, sputum, lower respiratory tract aspirates, bronchoalveolar lavage, nasopharyngeal wash/aspirate, or nasal aspirate.

RT-PCR test was conducted with the Gene Finder COVID-19 Plus Real Amp Kit (Elitech) or LightMix Modular SARS-Cov (Roche) according with the manufacturers' recommendations.

For all the patients eligible to the study, our reference standard was defined on the basis of the results of the RTPCR tests on specimens obtained on arrival at the ED. The reference was considered positive if at least one specimen tested positive for SARS-CoV-2.

Patients requiring hospitalization were temporarily isolated in a dedicated area of the ED, waiting for the test results. Patients who tested positive were transferred to a COVID-19-dedicated ward.
Upon a negative result on the first sample, the patient would ideally need to be retested after $48 \mathrm{~h}$. Patients with clinical features, laboratory results and imaging not suggestive of SARS-CoV-2 infection were retested and kept in the ED. Alternatively, when the clinical conditions were highly suggestive of COVID-19, the patients were immediately admitted to COVID-19-dedicated wards and kept in side rooms before the results of the first RT-PCR test were available, in order to avoid ED overcrowding.

It has wildly been documented that a single negative RT-PCR on respiratory specimens is insufficient to rule out COVID-19 [3-10]; hence, we performed two secondary analyses to assess the sensitivity of the study results to the reference standard. First, we excluded from the study cohort the subgroup of patients presenting with highly suspicious features of SARS-CoV-2 infection, who were tested on arrival at the ED and immediately hospitalized in COVID19-dedicated wards, and whose first RT-PCR test turned out to be negative. Most of these patients were ultimately classified as negative on the basis of a single negative test; thus, they are more likely to be misclassified at the reference standard than patients who were tested twice or who tested positive-the proportion of false positive RT-PCR tests has been documented to be very small [23]. Second, in the full study cohort, we considered a different reference standard, where we considered as positive the patients who had a positive specimen at the RT-PCR test (as in the primary analysis) or presented a CT scan suggestive of COVID-19, whenever the examination was performed.

\section{Statistical analysis}

The study results are presented following the recommendations of the Standards for Reporting of Diagnostic Accuracy (STARD) [24].

Quantitative variables are described with mean and standard deviation or median and interquartile range. Categorical and binary variables are presented as counts and proportions. The statistical analyses were performed with $\mathrm{R}$, version 3.6.1 [25].

The main analysis assessed the ability of the POCUS test to correctly classify patients with different results from the reference standard. The diagnostic accuracy of the POCUS test was primarily evaluated in terms of sensitivity and specificity and secondarily in terms of positive and negative predictive value (PPV and NPV) [24]. The estimates of these indicators are provided with $95 \%$ confidence intervals, computed with the exact binomial method [26]. The performances of the POCUS test were compared to the diagnostic accuracy of the chest X-ray, which was evaluated using the same methods. An identical methodological framework was used for the two secondary analyses. 


\section{Results}

\section{Study cohort}

A total of 515 patients visited the ED during the study period. The selection of the eligible patients $(384,74.6 \%)$ is presented in Fig. 1. Table 1 provides descriptive statistics of the enrolled patients. Four-hundred and one (77.9\%) patients underwent a bedside POCUS at the time of admission or shortly afterwards, which was systematically reported in the patient's records and suitable for the subsequent analysis.

Only 21 patients $(5.5 \%)$ underwent CT scan. The results were suggestive for COVID-19 for 17 (81.0\%) of them.

\section{Main analysis}

Of the 287 patients that tested positive at the reference standard, 23 had a negative result from the POCUS test (false negatives). The sensitivity of the test was $92.0 \%$ (95\% CI 88.2-94.9\%). Of the 97 patients classified as negative at the reference standard, 34 tested positive at the POCUS test (false positives). Therefore, the specificity of the test was $64.9 \%$ (95\% CI 54.6-74.4\%). With the observed prevalence of $74.7 \%$ (287/384), the PPV and NPV of the POCUS test were $88.6 \%$ (95\% CI 84.4-92.0) and $73.3 \%$ (95\% CI $62.6-82.2 \%$ ), respectively.

The chest X-ray result was not available on 11 patients (2.9\%), of whom 7 were positive at the reference standard. The X-ray showed 73 false negative and 41 false positive results. Therefore, sensitivity and specificity were $73.9 \%$

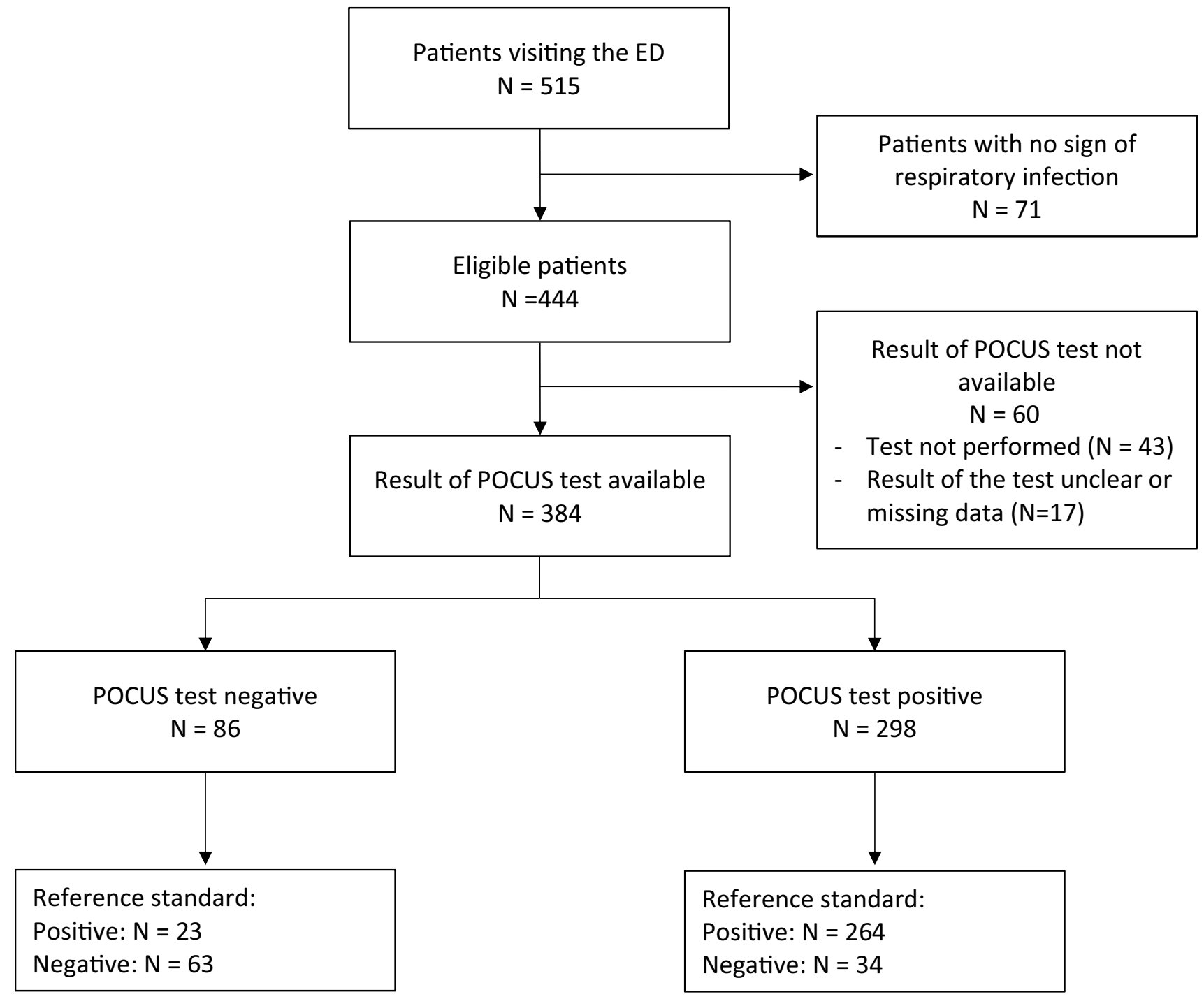

Fig. 1 Flow chart describing the patient selection and classification according to the POCUS test and reference standard 
Table 1 Demographic and clinical characteristics of the enrolled patients

\begin{tabular}{ll}
\hline Variables & $\begin{array}{l}\text { Enrolled } \\
\text { patients } \\
(N=384)\end{array}$ \\
\hline Age & \\
Mean (SD) & $65.4(17.1)$ \\
Median (Q1-Q3) & $68(53-79)$ \\
Missing & 1 \\
Sex (female)—N (\%) & $143(37.2)$ \\
Symptoms at ED presentation—N(\%) & \\
Cough & $257(66.9)$ \\
Dyspnea & $292(76.0)$ \\
Fever & $317(82.6)$ \\
Syncope & $20(5.2)$ \\
Vomit and/or diarrhea & $33(8.6)$ \\
CT scan- $N$ (\%) & \\
Suggestive of COVID-19 & $17(4.4)$ \\
Not suggestive of COVID-19 & $4(1.0)$ \\
Not executed & $363(94.5)$ \\
Chest X-ray- $N(\%)$ & \\
Suggestive of lung infection & $248(65.4)$ \\
Not suggestive of lung infection & $125(33.0)$ \\
Not executed & $6(1.6)$ \\
Missing & 5 \\
\hline
\end{tabular}

(95\% CI 68.4-79.0\%) and 55.9\% (95\% CI 45.2-66.2\%), considerably lower than those of the POCUS test. The PPV and NPV were $83.5 \%$ (95\% CI 78.2-87.9) and 41.6\% (95\% CI $32.9-50.8 \%$ ).

\section{Secondary analyses}

The patients who were hospitalized with a single negative RT-PCR test were 39 , i.e., $40.2 \%$ of the negative results at the reference standard. Thirty $(76.9 \%)$ tested positive at the POCUS test, while $9(23.1 \%)$ tested negative. After the exclusion of these patients, the specificity of the POCUS test raised to $93.1 \%$ (95\% CI 83.3-98.1\%). As we only excluded patients who were negative at the reference standard, the sensitivity of the test was unchanged. PPV and NPV, now with a prevalence of disease of $83.2 \%$ (287/345), were $98.5 \%$ (95\% CI 96.2\%-99.6\%) and 70.1\% (95\% CI 58.6-80.0\%), respectively. The chest X-ray was not available on 3 of the patients who were negative at the reference standard, after the exclusion of the subgroup. The false positives were 20 . Hence, the specificity of the chest X-ray was $63.6 \%(95 \%$ CI 49.6-76.2\%) on the selected cohort, while PPV and NPV were 91.2\% (95\% CI 86.7-94.5) and 32.4\% (95\% CI 23.7-42.1\%).

In the other secondary analysis, we considered the entire study cohort and classified as positive reference standard those patients with a positive RT-PCR test (as in the main analysis) or with a CT scan suggestive of COVID-19. Only 4 of the 17 positive CT scan examinations were classified as negative at the main reference standard (RT-PCR on respiratory specimens). Two of them (50\%) tested positive at the POCUS test. Using this modified reference, the sensitivity and specificity were $91.4 \%$ (95\% CI 87.6-94.4\%) and $65.6 \%$ (95\% CI 55.0-75.1\%). PPV and NPV were 89.3\% (95\% CI 85.2-92.5\%) and 70.9\% (95\% CI 60.1-80.2\%), respectively. The chest X-ray results were available on 3 of the 4 patients with negative reference standard and positive CT scan. Using the modified reference, the resulting sensitivity and specificity were $73.9 \%$ (95\% CI 68.3-78.9\%) and $56.7 \%$ (95\% CI 45.8-67.1\%), while the PPV and NPV were $84.3 \%$ (95\% CI 79.1-88.6\%) and 40.8\% (95\% CI 32.1-49.9\%).

\section{Ultrasound findings in patients with positive reference results}

Of the 287 patients who tested positive at the reference standard, 265 (92.3\%) presented with interstitial lung pattern and irregular pleura. Bilateral lung involvement (interstitial syndrome) was found in 246 cases $(85.7 \%)$. Lung consolidations were described in 113 patients (39.4\%). Pleural effusion was very uncommon among the study population (24 patients, $8.4 \%$ ).

\section{Ultrasound findings in patients with negative reference results}

Among the 34 patients with negative RT-PCR and positive POCUS, 12 (35.3\%) had one or more repeated negative swabs. The other patients were not retested either because they died in ED or soon after admission or because of technical or logistic difficulties.

Ultrasound findings in patients with negative RT-PCR on respiratory specimens were similar to those who were found positive at the standard reference: 32 (94.1\%) presented with interstitial lung pattern and irregular pleura, which was bilateral in 27 cases $(79.4 \%)$. Ten patients had one or more subpleural consolidations (29.4\%), only in 3 cases a pleural effusion was detected $(8.8 \%)$. Of the latter, one patient was diagnosed with pancreatitis and 2 with acute decompensated heart failure.

Six patients presented with isolated interstitial lung pattern with no subpleural consolidations. Of those, three were diagnosed with decompensated hearth failure, one with acute exacerbation of COPD, one with lung cancer and one with Respiratory Syncytial Virus infection.

Despite a negative first swab, 26 of the 34 patients, presenting with suggestive clinical and ultrasound features, were admitted to side rooms in COVID-19-dedicated wards 
to allow further work up. One patient died in ED, before a second sample was taken.

Of the 7 patients who were admitted to COVID-19-free wards, two presented suspicious ultrasound features: one underwent a CT scan, showing choledocholithiasis and a small ground glass area in the lingula. The second underwent neither a second swab nor chest $\mathrm{CT}$ and was diagnosed with acute decompensated heart failure and pneumonia.

\section{Discussion}

The Coronaviridae family of viruses includes six previously known human-infecting coronaviruses, which are enveloped, non-segmented, positive-sense RNA viruses that are broadly distributed in humans and other mammals [27].

The novel SARS-CoV- 2 coronavirus is the seventh member of the Coronaviridae family known to infect humans. Despite a lower reported mortality as compared to previously described coronaviruses (SARS-CoV and MERS$\mathrm{CoV})$, SARS-CoV-2 is highly contagious, and could lead to severe clinical manifestations, especially in patients with predisposing factors (older age and the presence of comorbidities) [1]. Lung involvement is common and leads to the highest morbidity and mortality [28].

Imaging examination has a determinant role, not only in the early detection and diagnosis, as discussed in this study, but also in monitoring the clinical course of the patients. For example, characteristic findings on CT imaging, including multiple, patchy, ground-glass opacity, crazy-paving pattern, and consolidation shadows, mainly distributed in the lower lobes, in peripheral and subpleural areas of both lungs, have been widely documented in COVID-19 patients. However, CT scan cannot always be considered as a first screening tool for all patients, due to logistic, economic, and healthrelated factors.

The SARS-CoV-2 pandemic has led to significant ED overcrowding, posing several issues in terms of patients' timely diagnosis, management and workflow. In particular, the impelling need of an upfront identification of infected patients, to provide timely treatments and prevent the spread of the virus, has motivated the search of a quick, simple, and reliable screening tool.

POCUS has been previously described as a useful imaging method to identify lung involvement in a variety of clinical settings [17]. We demonstrate the feasibility as a first-line screening tool of lung POCUS on patients admitted to ED with symptoms suggestive of SARS-CoV-2 infection. Our study considered a large cohort, including all the patients who consecutively visited the ED with signs and symptoms of respiratory infection. We were able to assess the performance of the POCUS test in high-prevalence settings (74.7\% of the patients were positive to the RT-PCR test), during a regional COVID-19 outbreak that caused a massive inflow of patients. Therefore, we were able to assess the diagnostic performance of POCUS in conditions were such test would be most useful in practice.

Moreover, our data show that chest X-ray lacks sensitivity and specificity to detect COVID-19-associated interstitial lung involvement. As no etiological diagnosis can be inferred on the basis of chest X-ray results alone, we considered all the images compatible with lung infection as positive, irrespective of any specific feature suggestive of COVID-19. This could partially explain the low specificity and PPV of chest X-ray in our data set.

We demonstrated that, as compared to the reference standard, POCUS has a high sensitivity.

Nevertheless, in our data set, the specificity of POCUS remained moderately low, as ultrasound findings do not allow an etiological diagnosis per se. Despite that, in the setting of a pandemic, when the prevalence of the disease is very high, there is a strong concordance between positive RT-PCR and ultrasound findings.

In our study, ultrasound findings showed a characteristic pattern of interstitial lung syndrome, with irregular pleura, and subpleural consolidations, as previously reported [29].

This pattern was described in both subgroups of patients, either positive or negative at the reference standard. In particular, the presence of pleural effusion seems to be very uncommon among COVID-19 patients, and more suggestive of other etiologies (such as decompensated heart failure or pancreatitis). On the other hand, the presence of interstitial lung syndrome with irregular pleura and subpleural consolidations seems to have a high positive predictive value. These data warrant a prospective evaluation.

It has been demonstrated elsewhere that a single negative RT-PCR on respiratory specimen is not sufficient to rule out COVID-19 pneumonia [11]. In our secondary analysis, when patients with a single negative swab were excluded, the specificity of POCUS increased dramatically.

These results suggest that, during a pandemic, it is sensible to isolate patients with highly suggestive clinical features and ultrasound findings, irrespective of the first swab results.

Moreover, we may speculate that, in the context of a highly suggestive presentation, a positive ultrasound should prompt further and repeated investigations to definitively rule out SARS-CoV-2 infection. In particular, at least two negative results of RT-PCR on different respiratory specimen taken $48 \mathrm{~h}$ apart would be needed to confirm the absence of COVID-19 infection [30].

Our study has several limitations. First, it was conducted in a retrospective fashion. Second, our physicians did not follow a standardized way to report ultrasound findings. Thus, some records were incomplete or not eligible for accurate analysis. In particular, US report was unclear or missing only in 17 cases. Third, even though ultrasound is an 
operator-dependent imaging modality, the assessment of the interobserver agreement between operators, which has been evaluated in the previous studies [31], was made impossible by our retrospective study design. However, all the operators received the same training and were instructed to use the same 12-region model. Fourth, many of our patients underwent a single nasopharyngeal swab, even in the presence of suggestive symptoms and positive ultrasound findings. This was mainly due to the lack of shared guidelines at the beginning of the pandemic and the incredible work overload. Nevertheless, most of the POCUS suggestive of interstitial pneumonia in patients with first negative swabs, which we considered as false positive, would probably have been truly positive if the RT-PCR test had been repeated or chest CT performed. If proven prospectively, this could affect the management of those patients with highly suspicious clinical features but a single negative RT-PCR on a respiratory specimen. We believe that a cautious hospitalization in a COVID-19-dedicated ward and discharge with indications to domestic quarantine should be followed. Finally, ultrasound findings remain non-specific when considered out of a clinical context. Interstitial lung syndrome merely reflects the increased non-aerated-to-aerated ratio in the lung parenchyma and has multiple causes, pulmonary oedema being the most common. When the prevalence of a disease is extremely high, as during the outbreak of an infectious disease, the characteristics of patients may differ substantially from those observed in low-prevalence conditions. Therefore, the diagnosis should always consider the whole clinical picture and epidemiological context.

In summary, during SARS-CoV-2 pandemic, POCUS performed in patients presenting with symptoms suggestive of COVID-19 infection allows the prompt identification of interstitial lung involvement. This dramatically affects the patients' management in terms of identification of those who require hospitalization, initiation of ventilation and other therapies, and cohort isolation of presumptive positive cases.

Author contributions $\mathrm{PB}, \mathrm{EG}, \mathrm{PF}, \mathrm{MP}, \mathrm{PG}$ and $\mathrm{AR}$ were responsible of data collection. FC, CS, MF, PB, EG, PF, MP, PG and AR reviewed the imaging results. GN and GB were responsible of the statistical analyses. FC, CS, GN, GB and MF drafted the manuscript. All authors substantially contributed to its revision and approved the final version.

Funding The authors received no specific funding for this work.

Availability of data and material The aggregated data that are necessary to reproduce the results of the statistical analyses are provided as Electronic Supplementary Material.

\section{Compliance with ethical standards}

Conflict of interest The authors declare that they have no conflict of interest.
Research involving human participants and/or animals Ethical approval for the study was provided by the institutional review board of the Area 1, Milan.

Informed consent As authorized by the institutional review board, the retrospective study did not require informed consent, in compliance with the Authorisation no. 9/2016-General Authorisation to Process Personal Data for Scientific Research Purposes.

Code availability We provide the $\mathrm{R}$ code and the raw output of the software as Electronic Supplementary Material.

\section{References}

1. Wang D, Hu B, Hu C, Zhu F, Liu X, Zhang J, Peng Z (2020) Clinical characteristics of 138 hospitalized patients with 2019 novel coronavirus-infected pneumonia in Wuhan, China. JAMA 323(11):1061-1069. https://doi.org/10.1001/jama.2020.1585

2. WHO. (n.d.) (2020) WHO Director-General's opening remarks at the media briefing on COVID-19-11. https://www.who.int/dg/ speeches/detail/who-director-general-s-opening-remarks-at-themedia-briefing-on-covid-19. Accessed 11 Mar 2020

3. Ai T, Yang Z, Hou H, Zhan C, Chen C, Lv W, Xia L (2020) Correlation of chest $\mathrm{CT}$ and RT-PCR testing in coronavirus disease 2019 (COVID-19) in China: a report of 1014 cases. Radiology. https://doi.org/10.1148/radiol.2020200642

4. Jin Y-H, Cai L, Cheng Z-S, Cheng H, Deng T, Fan Y-P, for the Zhongnan Hospital of Wuhan University Novel Coronavirus Management and Research Team, Evidence-Based Medicine Chapter of China International Exchange and Promotive Association for Medical and Health Care (CPAM) (2020) A rapid advice guideline for the diagnosis and treatment of 2019 novel coronavirus (2019-nCoV) infected pneumonia (standard version). Milit Med Res 7(1):4. https://doi.org/10.1186/s40779-020-0233-6

5. Li D, Wang D, Dong J, Wang N, Huang H, Xu H, Xia C (2020) False-negative results of real-time reverse-transcriptase polymerase chain reaction for severe acute respiratory syndrome coronavirus 2: role of deep-learning-based CT diagnosis and insights from two cases. Korean J Radiol 21(4):505-508. https://doi. org/10.3348/kjr.2020.0146

6. Fang Y, Zhang H, Xie J, Lin M, Ying L, Pang P, Ji W (2020) Sensitivity of chest CT for COVID-19: comparison to RT-PCR. Radiology. https://doi.org/10.1148/radiol.2020200432

7. Qian G-Q, Chen X-Q, Lv D-F, Ma AHY, Wang L-P, Yang N-B, Chen X-M (2020) Duration of SARS-CoV-2 viral shedding during COVID-19 infection. Infect Dis. https://doi.org/10.1080/23744 235.2020.1748705

8. Jiang G, Ren X, Liu Y, Chen H, Liu W, Guo Z, Shan H (2020) Application and optimization of RT-PCR in diagnosis of SARS-CoV-2 infection. Medrxiv. https://doi. org/10.1101/2020.02.25.20027755

9. Wang W, Xu Y, Gao R, Lu R, Han K, Wu G, Tan W (2020) Detection of SARS-CoV-2 in different types of clinical specimens. JAMA 323(18):1843-1844. https://doi.org/10.1001/ jama.2020.3786

10. Wölfel R, Corman VM, Guggemos W, Seilmaier M, Zange S, Müller MA, Wendtner C (2020) Virological assessment of hospitalized patients with COVID-2019. Nature. https://doi. org/10.1038/s41586-020-2196-x

11. Kim H, Hong H, Yoon SH (2020) Diagnostic performance of $\mathrm{CT}$ and reverse transcriptase-polymerase chain reaction for coronavirus disease 2019: a meta-analysis. Radiology. https://doi. org/10.1148/radiol.2020201343 
12. Dong D, Tang Z, Wang S, Hui H, Gong L, Lu Y, Li H (2020) The role of imaging in the detection and management of COVID-19: a review. In: IEEE reviews in biomedical engineering, 1-1. Presented at the IEEE reviews in biomedical engineering. https://doi. org/10.1109/RBME.2020.2990959

13. Zu ZY, Jiang MD, Xu PP, Chen W, Ni QQ, Lu GM, Zhang LJ (2020) Coronavirus disease 2019 (COVID-19): a perspective from China. Radiology. https://doi.org/10.1148/radiol.2020200490

14. Borghesi A, Maroldi R (2020) COVID-19 outbreak in Italy: experimental chest X-ray scoring system for quantifying and monitoring disease progression. Radiol Med (Torino) 125(5):509-513. https ://doi.org/10.1007/s11547-020-01200-3

15. de Barry O, Obadia I, Hajjam ME, Carlier R-Y, Obadia I, Hajjam ME, Carlier R-Y (2020) Chest-X-ray is a mainstay for follow-up in critically ill patients with covid-19 induced pneumonia. Eur J Radiol. https://doi.org/10.1016/j.ejrad.2020.109075

16. Lichtenstein D, Malbrain M (2019) Lung ultrasound in the critically ill (LUCI): a translational discipline. Anaesthesiol Intensive Ther 49(5):430-436. https://doi.org/10.5603/AIT.a2017.0063

17. Soldati G, Demi M (2017) The use of lung ultrasound images for the differential diagnosis of pulmonary and cardiac interstitial pathology. J Ultrasound 20(2):91-96. https://doi.org/10.1007/ s40477-017-0244-7

18. Volpicelli G, Gargani L (2020) Sonographic signs and patterns of COVID-19 pneumonia. Ultrasound J 12(1):22. https://doi. org/10.1186/s13089-020-00171-w

19. Mario Negri Institute for Pharmacological Research (2020) ECCOVID-PCS - early CPAP in COVID patients with respiratory failure. A prospective cohort study (Clinical trial registration no. NCT04323878). clinicaltrials.gov. https://clinicaltrials.gov/ct2/ show/NCT04323878. Accessed 27 May 2020

20. Via G, Storti E, Gulati G, Neri L, Mojoli F, Braschi A (2012) Lung ultrasound in the ICU: from diagnostic instrument to respiratory monitoring tool. Minerva Anestesiol 78(11):1282-1296

21. Volpicelli G, Elbarbary M, Blaivas M, Lichtenstein DA, Mathis G, Kirkpatrick AW, International Liaison Committee on Lung Ultrasound (ILC-LUS) for the International Consensus Conference on Lung Ultrasound (ICC-LUS) (2012) International evidence-based recommendations for point-of-care lung ultrasound. Intensive Care Med 38(4):577-591. https://doi.org/10.1007/s0013 4-012-2513-4

22. Vetrugno L, Bove T, Orso D, Barbariol F, Bassi F, Boero E, Kong R (2020) Our Italian experience using lung ultrasound for identification, grading and serial follow-up of severity of lung involvement for management of patients with COVID-19. Echocardiography 37(4):625-627. https://doi.org/10.1111/echo.14664

23. Corman VM, Landt O, Kaiser M, Molenkamp R, Meijer A, Chu DK, Drosten C (2020) Detection of 2019 novel coronavirus (2019$\mathrm{nCoV}$ ) by real-time RT-PCR. Eurosurveillance 25(3):2000045. https://doi.org/10.2807/1560-7917.ES.2020.25.3.2000045

24. Bossuyt PM, Reitsma JB, Bruns DE, Gatsonis CA, Glasziou PP, Irwig L, Cohen JF (2015) STARD 2015: an updated list of essential items for reporting diagnostic accuracy studies. BMJ. https:// doi.org/10.1136/bmj.h5527

25. R Core Team (2019) R: a language and environment for statistical computing. R Foundation for Statistical Computing, Vienna. https ://www.R-project.org/. Accessed 27 May 2020

26. Agresti A (2002) Categorical data analysis. Wiley, New York

27. Richman DD, Whitley RJ, Hayden FG (2016) Clinical virology. ASM Press, Washington, DC

28. Fu L, Wang B, Yuan T, Chen X, Ao Y, Fitzpatrick T, Zou H (2020) Clinical characteristics of coronavirus disease 2019 (COVID-19) in China: a systematic review and meta-analysis. J Infect. https:// doi.org/10.1016/j.jinf.2020.03.041

29. Buonsenso D, Piano A, Raffaelli F, Bonadia N, de Gaetano Donati K, Franceschi F (2020) Point-of-care lung ultrasound findings in novel coronavirus disease-19 pneumoniae: a case report and potential applications during COVID-19 outbreak. Eur Rev Med Pharmacol Sci 24(5):2776-2780. https://doi.org/10.26355/eurre v_202003_20549

30. Alfaraj SH, Al-Tawfiq JA, Memish ZA (2019) Middle East respiratory syndrome coronavirus intermittent positive cases: Implications for infection control. Am J Infect Control 47(3):290-293. https://doi.org/10.1016/j.ajic.2018.08.020

31. Razek AAKA, El-Basyouni SR (2016) Ultrasound of knee osteoarthritis: interobserver agreement and correlation with Western Ontario and McMaster Universities Osteoarthritis. Clin Rheumatol 35(4):997-1001. https://doi.org/10.1007/s10067-015-2990-2

Publisher's Note Springer Nature remains neutral with regard to jurisdictional claims in published maps and institutional affiliations. 